\title{
Tomato Domestication Affects Potential Functional Molecular Pathways of Root-Associated Soil Bacteria
}

\author{
Lisanne Smulders ${ }^{1}{ }^{(}$, Emilio Benítez ${ }^{1, *}$, Beatriz Moreno ${ }^{1}$, Álvaro López-García ${ }^{2}{ }^{\circledR}$, María J. Pozo ${ }^{2}{ }^{(0}$, \\ Victoria Ferrero ${ }^{3}$, Eduardo de la Peña ${ }^{4,5}$ (D) and Rafael Alcalá Herrera ${ }^{1}$ (D) \\ 1 Department of Environmental Protection, Estación Experimental del Zaidín (EEZ), CSIC, C/Profesor \\ Albareda 1, 18008 Granada, Spain; lisanne.smulders@eez.csic.es (L.S.); beatriz.moreno@eez.csic.es (B.M.); \\ rafa.alcala@eez.csic.es (R.A.H.) \\ 2 Department of Soil Microbiology and Symbiotic Systems, Estación Experimental del Zaidín (EEZ), CSIC, \\ C/Profesor Albareda 1, 18008 Granada, Spain; alvaro.lopez@eez.csic.es (Á.L.-G.); \\ mariajose.pozo@eez.csic.es (M.J.P.) \\ 3 Department of Biodiversity and Environmental Management, Campus de Vegazana s/n, University of León, \\ 24071 León, Spain; vferrv@unileon.es \\ 4 Finca Experimental "La Mayora" CSIC, Instituto de Hortofruticultura Subtropical y \\ Mediterránea (IHSM-UMA), CSIC, Algarrobo, 29750 Málaga, Spain; Eduardo.DeLaPena@ugent.be \\ 5 Department of Plants and Crops, Faculty of Bioscience Engineering, Ghent University, Coupure Links 653, \\ 9000 Ghent, Belgium \\ * Correspondence: emilio.benitez@eez.csic.es
}

\section{check for} updates

Citation: Smulders, L.; Benítez, E.; Moreno, B.; López-García, Á.; Pozo, M.J.; Ferrero, V.; de la Peña, E.; Alcalá Herrera, R. Tomato Domestication Affects Potential Functional Molecular Pathways of Root-Associated Soil Bacteria. Plants 2021, 10, 1942. https://doi.org/ 10.3390/plants10091942

Academic Editors: Petronia Carillo, Paula Baptista and Milan S. Stankovic

Received: 3 August 2021

Accepted: 14 September 2021

Published: 17 September 2021

Publisher's Note: MDPI stays neutral with regard to jurisdictional claims in published maps and institutional affiliations.

Copyright: (c) 2021 by the authors. Licensee MDPI, Basel, Switzerland. This article is an open access article distributed under the terms and conditions of the Creative Commons Attribution (CC BY) license (https:// creativecommons.org/licenses/by/ $4.0 /)$.

\begin{abstract}
While it has been well evidenced that plant domestication affects the structure of the rootassociated microbiome, there is a poor understanding of how domestication-mediated differences between rhizosphere microorganisms functionally affect microbial ecosystem services. In this study, we explore how domestication influenced functional assembly patterns of bacterial communities in the root-associated soil of 27 tomato accessions through a transect of evolution, from plant ancestors to landraces to modern cultivars. Based on molecular analysis, functional profiles were predicted and co-occurrence networks were constructed based on the identification of co-presences of functional units in the tomato root-associated microbiome. The results revealed differences in eight metabolic pathway categories and highlighted the influence of the host genotype on the potential functions of soil bacterial communities. In general, wild tomatoes differed from modern cultivars and tomato landraces which showed similar values, although all ancestral functional characteristics have been conserved across time. We also found that certain functional groups tended to be more evolutionarily conserved in bacterial communities associated with tomato landraces than those of modern varieties. We hypothesize that the capacity of soil bacteria to provide ecosystem services is affected by agronomic practices linked to the domestication process, particularly those related to the preservation of soil organic matter.
\end{abstract}

Keywords: bacterial functions; co-presence networks; metagenomics; microbial ecology; plant domestication

\section{Introduction}

The coevolutionary framework for analyzing interactions between plants and soil microorganisms has mainly been used for organisms involved in rhizosphere processes. Given that rhizosphere microbiomes are part of complex food webs affecting large numbers of nutrients released by the plant, it has been suggested that plants attract and select beneficial microbiomes by first releasing signals and then filtering species [1,2]. Rhizopshere microbiota are well known to play a critical role in both the adaptation of plants to the environment, but also contribute to a wide range of essential ecosystem services, such as carbon and nutrient cycling, plant growth promotion, soil structure stability, food web 
interactions and soil-atmosphere gas exchange, which ultimately affect soil productivity and sustainability [3].

In addition to plant genetics and developmental stage [4,5], other factors including soil management, agronomic practices, pathogen presence, soil $\mathrm{pH}$, nutrient content, and moisture, have been suggested to affect root microbial community structure [6-8]. However, the question of how the host and its environment regulate microbiome assembly and cooccurrence in plant species has not been addressed yet. This is of particular interest for crops in the context of plant-soil feedback, where plants can change soil biology and chemistry in ways that could affect subsequent plant growth [9].

Crop genetic diversity is usually reduced during plant domestication, which is associated with the selection of certain morphological traits such as root architecture and exudate composition, leading to striking differences between crops and their wild relatives $[10,11]$. Therefore, domestication is expected to have a direct impact on the type and diversity of below-ground microorganisms [9]. Indeed, domestication and genetic selection have progressively differentiated the microbiota of modern crops from those of their wild progenitors. It has also been postulated that crops are more likely to display negative feedbacks as compared to wild relatives, as domestication potentially disrupted beneficial rhizosphere associations [12]. Previous studies of cultivated plants evidenced differences between bacteria associated with differing plant genotypes such as wheat (Triticum aestivum L.), rice (Oryza sativa L.), barley (Hordeum vulgare L.) and tomato (Solanum lycopersicum Mill.) [13-15], suggesting that traits selected during domestication could have a significant influence on rhizosphere microbiota composition.

Although the structure of root-associated microbial communities is widely accepted to depend, to a greater or lesser degree, on the plant genotype, little is known about whether domestication-mediated differences between rhizosphere microorganisms functionally affect microbial ecosystem services. In this scenario, an evaluation of functional soil microbial genes could help to determine the effect of domestication on functional redundancy or cooccurrence of basic metabolic capacity in the rhizospheres of crop varieties and their wild ancestors [16]. This is essential to identify agricultural practices that resulted in reduced trade-offs between agricultural productivity and the provision of ecosystem services.

This study aims to explore how plant domestication influences the assembly patterns of soil microbial communities by metagenomic analysis of bacterial communities and predicted functions in the rhizosphere of different tomato varieties along a domestication gradient.

\section{Results}

\subsection{Bacterial Community Structure}

Figure 1 shows the relative bacterial abundance of the tomato root-associated soils based on the $16 \mathrm{~S}$ rRNA gene. Two main bacterial classes, Alphaproteobacteria and Actinobateria, dominated the total bacterial community with no differences observed between plant groups. Minority phyla such as Acidobacteria $(F=7.152, p=0.002)$ and Gemmatimonadetes $(F=4.720, p=0.013)$ were significantly less represented in the rhizosphere of wild tomato species than in tomato landraces and modern commercial cultivars. At the family level, the relative abundance of the Gemmatimonadaceae $(F=4.133, p=0.022)$, Microbacteriaceae $(F=5.419, p=0.007)$, and Streptomycetaceae $(F=4.752, p=0.022)$ families decreased, while Sphingomonadaceae ( $F=7.887, p=0.001$ ) increased in wild tomato relatives. Again, no differences between tomato landraces and modern commercial cultivars were detected.

The relative abundances of Acidobacteria_Gp16_unclassified $(F=3.701, p=0.031)$, Hyphomicrobiaceae ( $F=6.736, p=0.002)$, and Nocardioidaceae $(F=4.179, p=0.021)$ were different between wild and commercial cultivars, while landraces had intermediate values, generally not differing from the other two groups. 


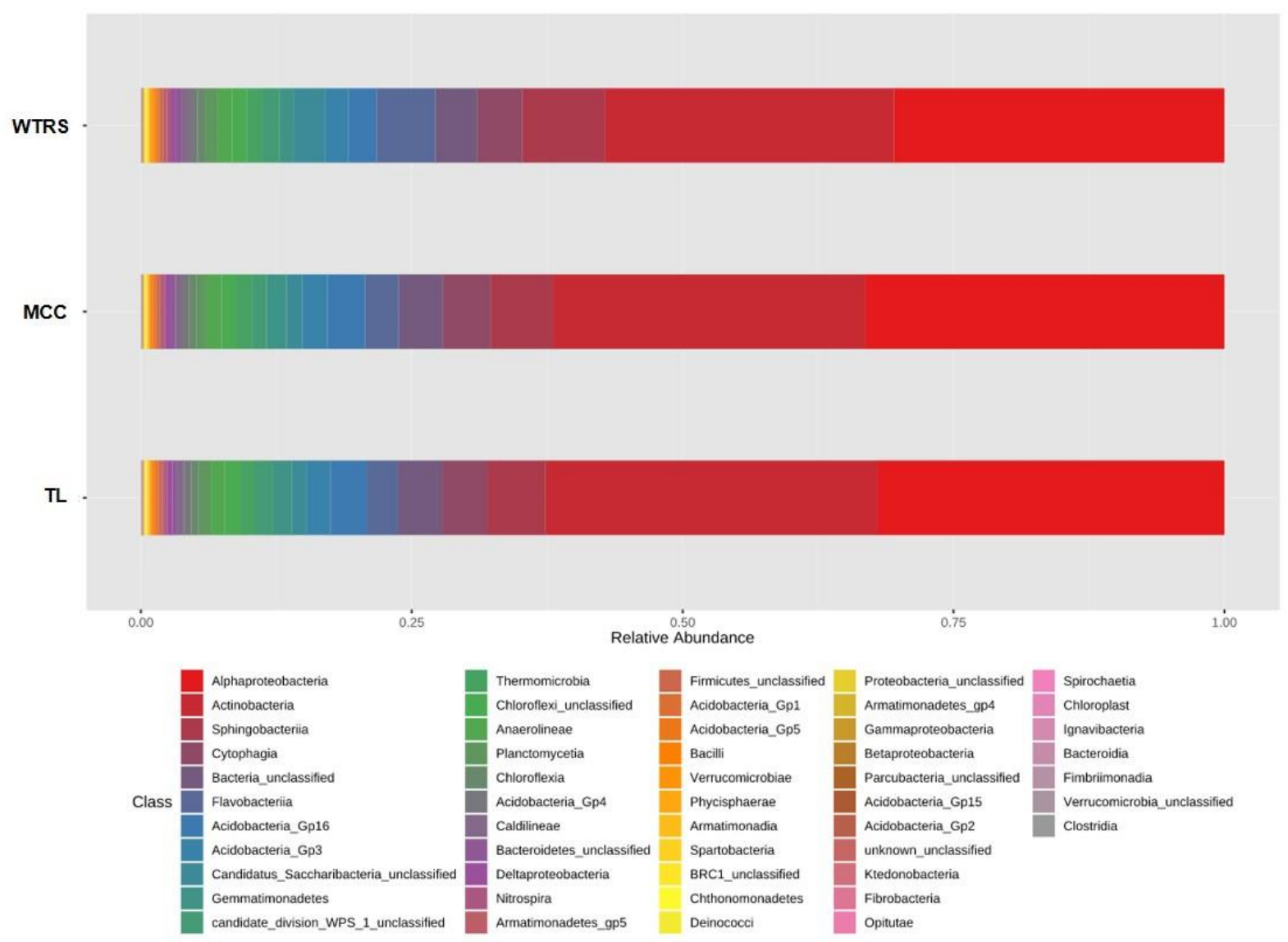

Figure 1. Relative abundance of bacteria of tomato rhizosphere soils. WTRS: wild tomato related species; TL: tomato landraces; MCC: modern commercial cultivars.

Linear discriminant analysis (LDA) at the genus level showed Pedobacter (Sphingobacteriaceae), Rodococcus, Skermanella and the proteobacterium Microvirga to be mainly responsible for the differences between the three tomato clusters (Figure 2). In addition, minor changes in bacterial diversity were observed at the OTU level (Table 1), as indicated by a significant decrease in the evenness of crop wild relatives $(F=6.623, p=0.003)$.

a)

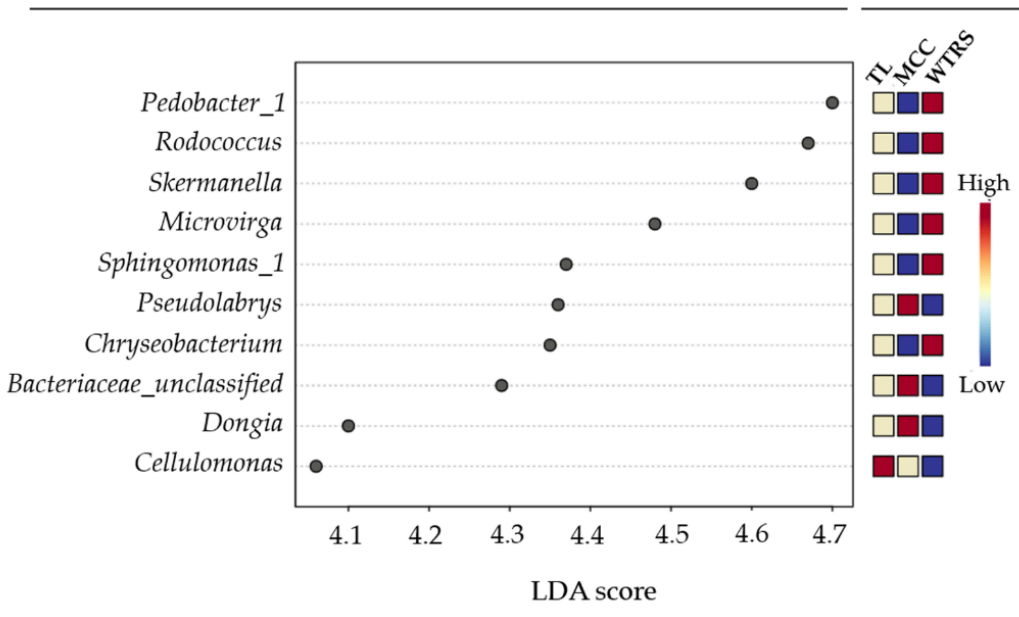

Figure 2. (a) Linear discriminant analysis (LDA) scores and, (b) heatmap from blue (low) via white to red (high) of genus relative abundances in root-associated soil of wild tomato related species (WTRS), tomato landraces (TL), and modern commercial cultivars (MCC). 
Table 1. Richness estimates and diversity indices (means \pm SE) for $16 \mathrm{~S}$ rRNA libraries of tomato rhizosphere soils. Different letters indicate a significant difference among tomato varieties $(p<0.05$, ANOVA, Dunn's post hoc-Bonferroni corrected $p$ values) when exist. WTRS: wild tomato related species; TL: tomato landraces; MCC: modern commercial cultivars.

\begin{tabular}{cccc}
\hline & WTRS & TL & MCC \\
\hline Shannon-Wiener Diversity Index & $5.79 \pm 0.26$ & $6.23 \pm 0.06$ & $6.18 \pm 0.09$ \\
Shannon Entropy & $8.35 \pm 0.37$ & $8.98 \pm 0.08$ & $8.92 \pm 0.12$ \\
Species Richness & $3260 \pm 346$ & $3727 \pm 155$ & $3471 \pm 231$ \\
Total Abundance & $52,838 \pm 3338$ & $59,144 \pm 1702$ & $57,100 \pm 2964$ \\
Simpson Diversity Index & $0.031 \pm 0.013$ & $0.010 \pm 0.001$ & $0.011 \pm 0.002$ \\
Evenness & $0.719 \pm 0.021 \mathrm{~b}$ & $0.759 \pm 0.004 \mathrm{a}$ & $0.764 \pm 0.005 \mathrm{a}$ \\
Chao-1 & $4453 \pm 450$ & $4983 \pm 241$ & $4619 \pm 329$ \\
\hline
\end{tabular}

\subsection{Bacterial Community Functional Analysis}

We used metagenomics analysis to predict the functional potential of the bacterial community and to explore associated metabolic pathway networks using Kyoto Encyclopedia of Gene and Genome (KEGG) clusters.

At the level of functional units of gene sets, all tomato varieties shared all the 181 predicted functions related to soil bacteria (Table S1). However, 68 of them differed among tomato domestication types (Table 2). In general, wild tomatoes differed from modern cultivars and tomato landraces that usually showed similar values, but generally tomato landraces had intermediate values between modern cultivars and wild relatives. For example, the levels of the aromatic degradation metabolic pathway category, except for module M00541 (benzoyl-CoA degradation), tended to be significantly higher in bacteria growing in wild tomato accessions, indicating that tomato landraces drive bacterial communities with similar levels of predicted functions as modern commercial cultivars. Similarly, while the values for the metabolic categories of nitrogen, sulfur, cofactor/vitamin and purine were decreased in modern cultivars with respect to wild varieties, no differences between these wild and landrace cultivars were detected. By contrast, lipopolysaccharide and lipid metabolic pathway levels were clearly higher in both landrace and modern cultivars with respect to their wild relatives.

Table 2. Functional units of gene sets in metabolic pathways (KEGG modules) of tomato rhizosphere soils differentially represented among tomato varieties. Different letters indicate a significant difference among tomato varieties (ANOVA, Dunn's post hoc-Bonferroni corrected $p$ values). WTRS: wild tomato related species; TL: tomato landraces; MCC: modern commercial cultivars.

\begin{tabular}{|c|c|c|c|c|c|}
\hline Pathway Modules & & WTRS & TL & MCC & $p$-Value \\
\hline \multirow{6}{*}{$\begin{array}{l}\text { Amino acid metabolism; } \\
\text { Arginine and proline } \\
\text { metabolism }\end{array}$} & $\begin{array}{l}\text { M00015_Proline biosynthesis, glutamate } \\
=>\text { proline }\end{array}$ & $1656 a$ & $1609 b$ & $1626 b$ & 0.02086 \\
\hline & $\begin{array}{l}\text { M00023_Tryptophan biosynthesis, chorismate } \\
=>\text { tryptophan }\end{array}$ & $3173 b$ & $3234 a$ & $3205 a$ & 0.008801 \\
\hline & $\begin{array}{l}\text { M00037_Melatonin biosynthesis, tryptophan } \\
=>\text { serotonin }=>\text { melatonin }\end{array}$ & $69 \mathrm{~b}$ & $77 a$ & $74 \mathrm{ab}$ & 0.04182 \\
\hline & $\begin{array}{l}\text { M00040_Tyrosine biosynthesis, chorismate }=> \\
\text { arogenate }=>\text { tyrosine }\end{array}$ & $508 a$ & $464 b$ & $467 \mathrm{~b}$ & $5.579 \times 10^{-6}$ \\
\hline & $\begin{array}{l}\text { M00042_Catecholamine biosynthesis, tyrosine } \\
=>\text { dopamine }=>\text { noradrenaline }=>\text { adrenaline }\end{array}$ & $95 b$ & $106 a$ & $104 a b$ & 0.02289 \\
\hline & $\begin{array}{l}\text { M00533_Homoprotocatechuate degradation, } \\
\text { homoprotocatechuate }=> \\
\text { 2-oxohept-3-enedioate }\end{array}$ & $491 \mathrm{a}$ & $474 \mathrm{~b}$ & $473 \mathrm{ab}$ & 0.04715 \\
\hline $\begin{array}{l}\text { Amino acid metabolism; } \\
\text { Aromatic amino acid } \\
\text { metabolism }\end{array}$ & $\begin{array}{l}\text { M00545_Trans-cinnamate degradation, } \\
\text { trans-cinnamate }=>\text { acetyl-CoA }\end{array}$ & $1048 \mathrm{a}$ & $1004 b$ & $1014 b$ & 0.001853 \\
\hline
\end{tabular}


Table 2. Cont.

\begin{tabular}{|c|c|c|c|c|c|}
\hline Pathway Modules & & WTRS & TL & MCC & $p$-Value \\
\hline $\begin{array}{l}\text { Amino acid metabolism; } \\
\text { Branched-chain amino } \\
\text { acid metabolism }\end{array}$ & $\begin{array}{l}\text { M00036_Leucine degradation, leucine => } \\
\text { acetoacetate + acetyl-CoA }\end{array}$ & $7057 \mathrm{a}$ & $6938 b$ & $6874 b$ & $6.597 \times 10^{-5}$ \\
\hline \multirow{3}{*}{$\begin{array}{l}\text { Amino acid metabolism; } \\
\text { Cysteine and methionine } \\
\text { metabolism }\end{array}$} & $\begin{array}{l}\text { M00017_Methionine biosynthesis, apartate }=> \\
\text { homoserine }=>\text { methionine }\end{array}$ & $5393 b$ & $5464 a$ & $5422 \mathrm{a}$ & 0.006857 \\
\hline & M00035_Methionine degradation & $2031 b$ & $2101 \mathrm{a}$ & $2065 b$ & $6.836 \times 10^{-5}$ \\
\hline & $\begin{array}{l}\text { M00338_Cysteine biosynthesis, homocysteine + } \\
\text { serine => cysteine }\end{array}$ & $233 c$ & $276 a$ & $257 \mathrm{~b}$ & $9.38 \times 10^{-11}$ \\
\hline $\begin{array}{l}\text { Amino acid metabolism; } \\
\text { Lysine metabolism }\end{array}$ & $\begin{array}{l}\text { M00031_Lysine biosynthesis, mediated by } \\
\text { LysW, 2-aminoadipate => lysine }\end{array}$ & $81 b$ & $106 a$ & $98 b$ & $1.069 \times 10^{-5}$ \\
\hline \multirow{2}{*}{$\begin{array}{l}\text { Amino acid metabolism; } \\
\text { Other amino acid } \\
\text { metabolism }\end{array}$} & $\begin{array}{l}\text { M00118_Glutathione biosynthesis, glutamate } \\
\text { => glutathione }\end{array}$ & $944 a$ & $876 b$ & $880 \mathrm{~b}$ & $7.781 \times 10^{-8}$ \\
\hline & $\begin{array}{l}\text { M00027_GABA } \\
\text { (gamma-Aminobutyrate) shunt }\end{array}$ & $2018 \mathrm{a}$ & $1942 b$ & $1921 b$ & $1.037 \times 10^{-5}$ \\
\hline \multirow{3}{*}{$\begin{array}{l}\text { Amino acid metabolism; } \\
\text { Polyamine biosynthesis }\end{array}$} & $\begin{array}{l}\text { M00133_Polyamine biosynthesis, arginine }=> \\
\text { agmatine }=>\text { putrescine }=>\text { spermidine }\end{array}$ & $865 b$ & $895 a$ & $879 \mathrm{ab}$ & 0.01599 \\
\hline & $\begin{array}{l}\text { M00134_Polyamine biosynthesis, arginine => } \\
\text { ornithine => putrescine }\end{array}$ & $872 a$ & $838 b$ & $841 b$ & 0.008463 \\
\hline & $\begin{array}{l}\text { M00136_GABA biosynthesis, prokaryotes, } \\
\text { putrescine => GABA }\end{array}$ & $677 \mathrm{a}$ & $620 \mathrm{~b}$ & $636 b$ & $6.265 \times 10^{-9}$ \\
\hline $\begin{array}{l}\text { Amino acid metabolism; } \\
\text { Serine and threonine } \\
\text { metabolism }\end{array}$ & $\begin{array}{l}\text { M00555_Betaine biosynthesis, } \\
\text { choline => betaine }\end{array}$ & $1473 a$ & $1377 \mathrm{~b}$ & $1383 b$ & $4.206 \times 10^{-11}$ \\
\hline \multirow{7}{*}{$\begin{array}{l}\text { Carbohydrate metabolism; } \\
\text { Central carbohydrate } \\
\text { metabolism }\end{array}$} & $\begin{array}{l}\text { M00006_Pentose phosphate pathway, } \\
\text { oxidative phase, glucose } 6 \mathrm{P}=>\text { ribulose } 5 \mathrm{P}\end{array}$ & $1535 \mathrm{ab}$ & $1523 b$ & $1546 a$ & 0.04953 \\
\hline & M00077_Chondroitin sulfate degradation & $105 b$ & $118 \mathrm{a}$ & $123 a$ & $6.408 \times 10^{-5}$ \\
\hline & $\begin{array}{l}\text { M00008_Entner-Doudoroff pathway, } \\
\text { glucose- } 6 \mathrm{P}=>\text { glyceraldehyde- } 3 \mathrm{P}+\text { pyruvate }\end{array}$ & $1993 a$ & $1905 c$ & $1928 b$ & $2.838 \times 10^{-6}$ \\
\hline & M00009_Citrate cycle (TCA cycle, Krebs cycle) & $12,529 a$ & $12,667 \mathrm{~b}$ & $12,563 a$ & 0.0001383 \\
\hline & $\begin{array}{l}\text { M00011_Citrate cycle, second carbon oxidation, } \\
\text { 2-oxoglutarate => oxaloacetate }\end{array}$ & $9185 b$ & $9286 a$ & $9207 \mathrm{~b}$ & 0.001811 \\
\hline & $\begin{array}{l}\text { M00003_Gluconeogenesis, oxaloacetate => } \\
\text { fructose- } 6 \mathrm{P}\end{array}$ & $5474 b$ & $5544 a$ & $5498 b$ & 0.008293 \\
\hline & $\begin{array}{l}\text { M00633_Semi-phosphorylative } \\
\text { Entner-Doudoroff pathway, } \\
\text { gluconate/galactonate => glycerate-3P }\end{array}$ & $85 \mathrm{~b}$ & $91 \mathrm{ab}$ & $95 a$ & 0.03837 \\
\hline \multirow{7}{*}{$\begin{array}{l}\text { Carbohydrate metabolism; } \\
\text { Other carbohydrate } \\
\text { metabolism }\end{array}$} & $\begin{array}{l}\text { M00061_D-Glucuronate degradation, } \\
\text { D-glucuronate => pyruvate }+ \\
\text { D-glyceraldehyde 3P }\end{array}$ & $1694 a$ & $1654 b$ & $1680 \mathrm{ab}$ & 0.01455 \\
\hline & M00081_Pectin degradation & $113 b$ & $129 \mathrm{a}$ & $132 \mathrm{a}$ & $6.318 \times 10^{-5}$ \\
\hline & $\begin{array}{l}\text { M00114_Ascorbate biosynthesis, plants, } \\
\text { glucose-6P => ascorbate }\end{array}$ & $2958 \mathrm{ab}$ & $2995 a$ & $2949 b$ & 0.0271 \\
\hline & $\begin{array}{l}\text { M00131_Inositol phosphate metabolism, } \\
\text { Ins }(1,3,4,5) \mathrm{P} 4=>\text { Ins }(1,3,4) \mathrm{P} 3=>\text { myo-inositol }\end{array}$ & $1003 a$ & $969 b$ & $968 \mathrm{ab}$ & 0.02686 \\
\hline & $\begin{array}{l}\text { M00550_Ascorbate degradation, ascorbate }=> \\
\text { D-xylulose-5P }\end{array}$ & $27 \mathrm{a}$ & $19 b$ & $18 \mathrm{~b}$ & $1.195 \times 10^{-5}$ \\
\hline & $\begin{array}{l}\text { M00554_Nucleotide sugar biosynthesis, } \\
\text { galactose => UDP-galactose }\end{array}$ & $199 \mathrm{~b}$ & $207 a b$ & $217 \mathrm{a}$ & 0.005133 \\
\hline & $\begin{array}{l}\text { M00565_Trehalose biosynthesis, } \\
\text { D-glucose 1P => trehalose }\end{array}$ & $3380 \mathrm{~b}$ & $3603 a$ & $3666 a$ & $2.2 \times 10^{-16}$ \\
\hline
\end{tabular}


Table 2. Cont.

\begin{tabular}{|c|c|c|c|c|c|}
\hline Pathway Modules & & WTRS & TL & MCC & $p$-Value \\
\hline \multirow{5}{*}{$\begin{array}{l}\text { Energy metabolism; } \\
\text { Carbon fixation }\end{array}$} & $\begin{array}{l}\text { M00170_C4-dicarboxylic acid cycle, } \\
\text { phosphoenolpyruvate carboxykinase type }\end{array}$ & $1302 c$ & $1357 \mathrm{a}$ & $1321 \mathrm{bc}$ & $1.883 \times 10^{-5}$ \\
\hline & $\begin{array}{l}\text { M00172_C4-dicarboxylic acid cycle, } \\
\text { NADP_-malic enzyme type }\end{array}$ & $3505 a$ & $3444 b$ & $3445 b$ & 0.03007 \\
\hline & $\begin{array}{l}\text { M00173_Reductive citrate cycle } \\
\text { (Arnon-Buchanan cycle) }\end{array}$ & $10,778 b$ & $10,891 \mathrm{a}$ & $10,850 \mathrm{ab}$ & 0.004089 \\
\hline & M00374_Dicarboxylate-hydroxybutyrate cycle & $7259 b$ & $7345 a$ & $7333 a$ & 0.01178 \\
\hline & $\begin{array}{l}\text { M00620_Incomplete reductive citrate cycle, } \\
\text { acetyl-CoA => oxoglutarate }\end{array}$ & $2168 b$ & $2224 a$ & $2231 \mathrm{a}$ & 0.0007637 \\
\hline \multirow{7}{*}{$\begin{array}{l}\text { Energy metabolism;; } \\
\text { Methane metabolism }\end{array}$} & $\begin{array}{l}\text { M00344_Formaldehyde assimilation, } \\
\text { xylulose monophosphate pathway }\end{array}$ & $913 b$ & $944 a$ & $942 \mathrm{ab}$ & 0.03158 \\
\hline & $\begin{array}{l}\text { M00345_Formaldehyde assimilation, } \\
\text { ribulose monophosphate pathway }\end{array}$ & $749 \mathrm{~b}$ & $808 \mathrm{a}$ & $800 \mathrm{a}$ & $6.137 \times 10^{-7}$ \\
\hline & $\begin{array}{l}\text { M00346_Formaldehyde assimilation, } \\
\text { serine pathway }\end{array}$ & $3166 b$ & $3234 a$ & $3226 a b$ & 0.006593 \\
\hline & $\begin{array}{l}\text { M00356_Methanogenesis, } \\
\text { methanol => methane }\end{array}$ & $22 \mathrm{~b}$ & $26 a b$ & $27 a$ & 0.03877 \\
\hline & M00358_Coenzyme M biosynthesis & $177 \mathrm{~b}$ & 190a & $198 \mathrm{a}$ & 0.0004887 \\
\hline & M00378_F420 biosynthesis & $82 b$ & $93 a$ & $89 \mathrm{ab}$ & 0.05467 \\
\hline & $\begin{array}{l}\text { M00563_Methanogenesis, methy- } \\
\text { lamine/dimethylamine/trimethylamine } \\
\text { => methane }\end{array}$ & $465 a$ & $434 \mathrm{~b}$ & $464 \mathrm{a}$ & $3.724 \times 10^{-6}$ \\
\hline $\begin{array}{l}\text { Energy metabolism; } \\
\text { Nitrogen metabolism }\end{array}$ & $\begin{array}{l}\text { M00530_Dissimilatory nitrate reduction, } \\
\text { nitrate => ammonia }\end{array}$ & $1864 a$ & $1823 b$ & $1848 \mathrm{a}$ & 0.01764 \\
\hline $\begin{array}{l}\text { Energy metabolism; } \\
\text { Sulfur metabolism }\end{array}$ & $\begin{array}{l}\text { M00176_Assimilatory sulfate reduction, } \\
\text { sulfate => H2S }\end{array}$ & $2814 a$ & $2741 b$ & $2766 \mathrm{ab}$ & 0.006395 \\
\hline \multirow{4}{*}{$\begin{array}{l}\text { Glycan metabolism; } \\
\text { Glycosaminoglycan } \\
\text { metabolis }\end{array}$} & M00076_Dermatan sulfate degradation & $115 b$ & $129 \mathrm{a}$ & $135 a$ & $2.073 \times 10^{-5}$ \\
\hline & M00077_Chondroitin sulfate degradation & $105 b$ & $118 \mathrm{a}$ & $123 a$ & $6.408 \times 10^{-5}$ \\
\hline & M00078_Heparan sulfate degradation & $191 b$ & $215 a$ & $224 a$ & $2.272 \times 10^{-7}$ \\
\hline & M00079_Keratan sulfate degradation & $475 b$ & $526 a$ & $547 \mathrm{a}$ & $1.002 \times 10^{-12}$ \\
\hline \multirow{2}{*}{$\begin{array}{l}\text { Glycan metabolism; } \\
\text { Lipopolysaccharide } \\
\text { metabolism }\end{array}$} & $\begin{array}{l}\text { M00060_KDO2-lipid A biosynthesis, } \\
\text { Raetz pathway, LpxL-LpxM type }\end{array}$ & $3058 b$ & $3132 a$ & $3124 a$ & 0.001684 \\
\hline & $\begin{array}{l}\text { M00064_ADP-L-glycero-D-manno-heptose } \\
\text { biosynthesis }\end{array}$ & $692 b$ & $743 a$ & $771 \mathrm{a}$ & $3.151 \times 10^{-7}$ \\
\hline \multirow{3}{*}{$\begin{array}{l}\text { Lipid metabolism; } \\
\text { Fatty acid metabolism }\end{array}$} & M00082_Fatty acid biosynthesis, initiation & $3785 b$ & $3861 \mathrm{a}$ & $3842 \mathrm{ab}$ & 0.01467 \\
\hline & M00083_Fatty acid biosynthesis, elongation & $9121 b$ & $9218 \mathrm{a}$ & $9214 a$ & 0.01719 \\
\hline & M00086_beta-Oxidation, acyl-CoA synthesis & $1699 b$ & $1743 a$ & $1746 a b$ & 0.01575 \\
\hline $\begin{array}{l}\text { Lipid metabolism; } \\
\text { Lipid metabolism }\end{array}$ & M00113_Jasmonic acid biosynthesis & $428 b$ & $454 a$ & $438 \mathrm{~b}$ & 0.002276 \\
\hline \multirow{4}{*}{$\begin{array}{l}\text { Metabolism of cofactors } \\
\text { and vitamins; } \\
\text { Cofactor and vitamin } \\
\text { metabolism }\end{array}$} & $\begin{array}{l}\text { M00116_Menaquinone biosynthesis, } \\
\text { chorismate => menaquinol }\end{array}$ & $943 b$ & $1026 a$ & $977 \mathrm{~b}$ & $1.104 \times 10^{-10}$ \\
\hline & $\begin{array}{l}\text { M00117_Ubiquinone biosynthesis, } \\
\text { prokaryotes, chorismate => ubiquinone }\end{array}$ & $2772 a$ & $2703 b$ & $2707 \mathrm{ab}$ & 0.01037 \\
\hline & $\begin{array}{l}\text { M00122_Cobalamin biosynthesis, } \\
\text { cobinamide => cobalamin }\end{array}$ & $2143 a$ & $2105 b$ & $2153 a$ & 0.00244 \\
\hline & $\begin{array}{l}\text { M00128_Ubiquinone biosynthesis, eukaryotes, } \\
\text { 4-hydroxybenzoate => ubiquinone }\end{array}$ & $74 a$ & $64 \mathrm{~b}$ & $67 a b$ & 0.01119 \\
\hline $\begin{array}{l}\text { Nucleotide metabolism; } \\
\text { Purine metabolism }\end{array}$ & M00546_Purine degradation, xanthine => urea & $2126 a$ & $2089 b$ & $2125 a$ & 0.01079 \\
\hline
\end{tabular}


Table 2. Cont.

\begin{tabular}{|c|c|c|c|c|c|}
\hline Pathway Modules & & WTRS & TL & MCC & $p$-Value \\
\hline \multirow{7}{*}{$\begin{array}{l}\text { Xenobiotics } \\
\text { biodegradation; } \\
\text { Aromatics degradation }\end{array}$} & $\begin{array}{l}\text { M00537_Xylene degradation, } \\
\text { xylene => methylbenzoate }\end{array}$ & $215 a$ & $199 b$ & $200 \mathrm{ab}$ & 0.01542 \\
\hline & $\begin{array}{l}\text { M00541_Benzoyl-CoA degradation, } \\
\text { benzoyl-CoA => 3-hydroxypimeloyl-CoA }\end{array}$ & $59 \mathrm{~b}$ & $67 a$ & $67 a b$ & 0.02392 \\
\hline & $\begin{array}{l}\text { M00548_Benzene degradation, } \\
\text { benzene => catechol }\end{array}$ & $27 a$ & $20 \mathrm{~b}$ & $21 b$ & 0.0002254 \\
\hline & $\begin{array}{l}\text { M00551_Benzoate degradation, benzoate }=> \\
\text { catechol } / \text { methylbenzoate }=>\text { methylcatechol }\end{array}$ & $124 a$ & $108 b$ & $110 \mathrm{~b}$ & 0.003958 \\
\hline & $\begin{array}{l}\text { M00568_Catechol ortho-cleavage, catechol => } \\
\text { 3-oxoadipate }\end{array}$ & $445 a$ & $421 b$ & $433 \mathrm{ab}$ & 0.01165 \\
\hline & $\begin{array}{l}\text { M00569_Catechol meta-cleavage, catechol => } \\
\text { acetyl-CoA/4-methylcatechol => } \\
\text { propanoyl-CoA }\end{array}$ & $466 a$ & $441 b$ & $430 \mathrm{~b}$ & 0.001843 \\
\hline & $\begin{array}{l}\text { M00637_Anthranilate degradation, } \\
\text { anthranilate => catechol }\end{array}$ & $90 a$ & $73 b$ & $82 b$ & $1.726 \times 10^{-5}$ \\
\hline
\end{tabular}

Amino acid metabolism pathways exhibited no clear tendency, although in modern commercial cultivars, cysteine and methionine pathway levels were higher and those of other amino acid pathways were lower. A similar variable pattern was observed with respect to both central carbohydrate and other carbohydrate metabolic pathways in the category of carbohydrate metabolism.

Finally, a marked increase in the carbon fixation and methane metabolic subfunctions and in the metabolic pathway categories glycan metabolism and lipid metabolism, respectively, was observed in the modern commercial cultivars.

\subsection{Functional Networks of KEGG Orthologous Groups}

Figure 3 and Table 3 show the co-presence networks and the topological properties of functional networks, respectively, for the modern:wild, landraces:wild and modern:landraces pairs. An increase in the average number of neighbors and a decrease in the characteristic path length were found in landraces:wild pairs (Table 3). Additionally, an increase in the network radius and diameter were detected in the pair modern:landraces. Finally, the pair modern:wild showed the largest number of KEGG-module nodes and the largest number of edges or inter-node connections in the network. The clustering coefficient, which reflects the tendency of organisms to form relatively high-density clusters, was zero. Co-occurrence networks are generated by connecting pairs of terms using a set of criteria defining co-occurrence. These networks connect across, rather than between, nodes. Every node, in which none of whose neighbors connect to each other, has a clustering coefficient of zero.

Highly connected clusters were retrieved for every network, four for the pair modern:wild and three for the other two pairs (Figures 4-6). On a closer analysis, we detected some links in highly connected clusters. Thus, bacterial functional units M00026 (histidine biosynthesis, PRPP $=>$ histidine), M00032 (lysine degradation, lysine $=>$ saccharopine $=>$ acetoacetyl-CoA), M00141 (C1-unit interconversion) and M00376 (3-hydroxypropionate bicycle) were highly connected in the soil of tomato landraces and wild relatives (Figure 4a), whereas modern varieties and wild relatives were connected by modules M00141, M00376, M00021 (cysteine biosynthesis, serine $=>$ cysteine) and M00089 (triacylglycerol biosynthesis) (Figure 5a-c). Finally, modules M00026 and M00141 were highly represented in modern varieties and tomato landraces (Figure $6 a, b)$. 
a)

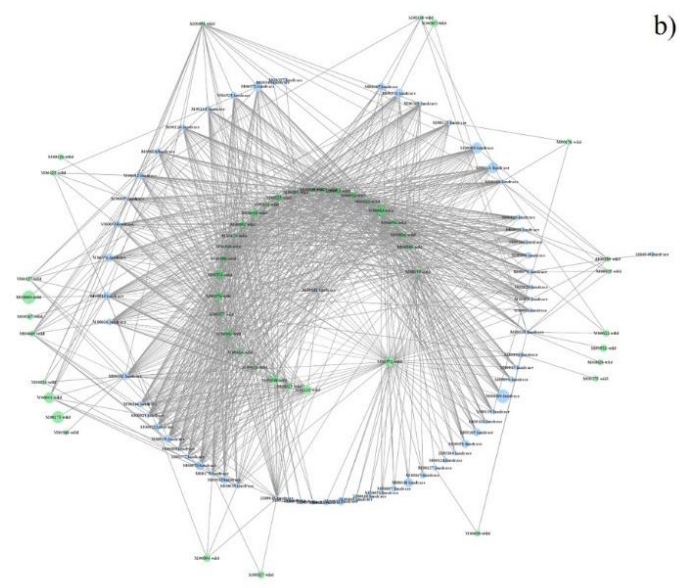

c)

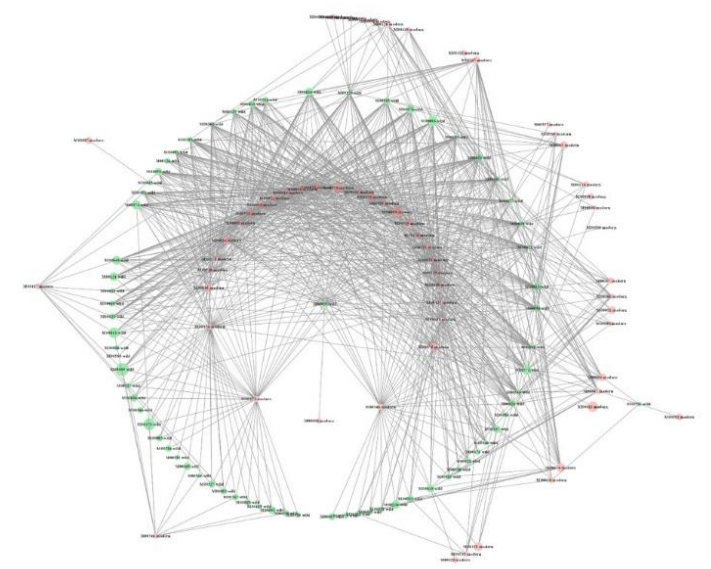

b)

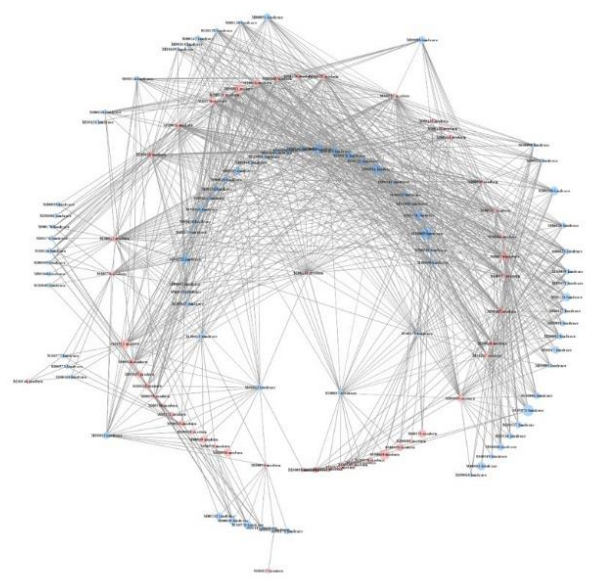

Figure 3. Co-presence network for the couples: (a) tomato landraces:wild tomato related species, (b) modern commercial cultivars:tomato landraces, (c) modern commercial cultivars:wild tomato related species. Node sizes reflect average relative abundance of each KEGG module. The line thickness is proportional to the edge weight. Node colors: green for wild tomato related species, blue for tomato landraces and red for modern commercial cultivars.

Table 3. Topological properties of pairwise functional networks. WTRS: wild tomato related species; TL: tomato landraces; MCC: modern commercial cultivars.

\begin{tabular}{cccc}
\hline & MCC:WTRS & TL:WTRS & MCC:TL \\
\hline Number of nodes & 133 & 116 & 132 \\
Number of edges & 1005 & 1003 & 1001 \\
Average number of neighbors & 15,113 & 17,293 & 15,167 \\
Network diameter & 6 & 6 & 7 \\
Network radius & 3 & 3 & 4 \\
Characteristic path length & 2.542 & 2.371 & 2.577 \\
Clustering coefficient & 0.000 & 0.000 & 0.000 \\
Network density & 0.114 & 0.150 & 0.116 \\
Network heterogeneity & 0.850 & 0.780 & 0.869 \\
Network centralization & 0.230 & 0.325 & 0.309 \\
Connected components & 1 & 1 & 1 \\
\hline
\end{tabular}


a)

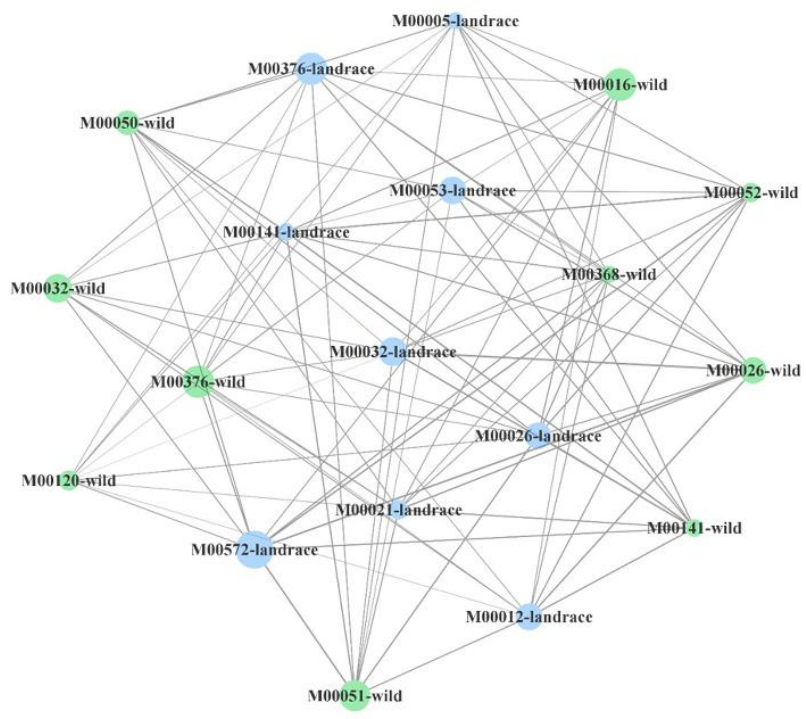

b)

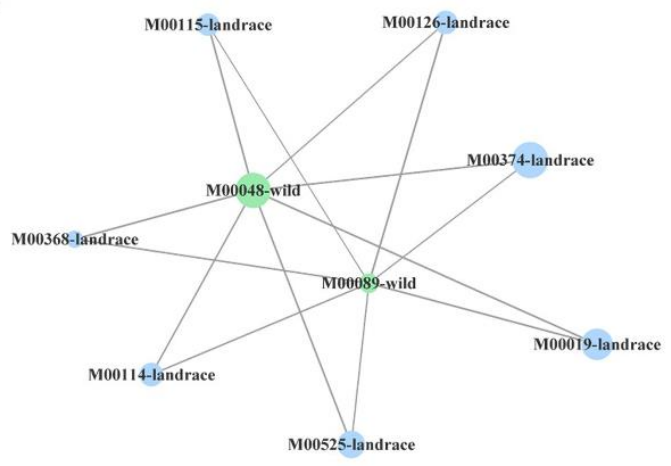

c)

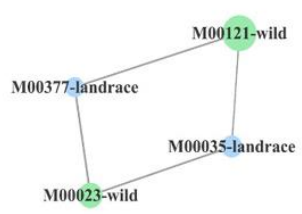

Figure 4. Three $(\mathbf{a}-\mathbf{c})$ most connected clusters in co-presence networks for the couple tomato landraces (blue): wild tomato related species (green). Node sizes reflect average relative abundance of each KEGG module. The line thickness is proportional to the edge weight.

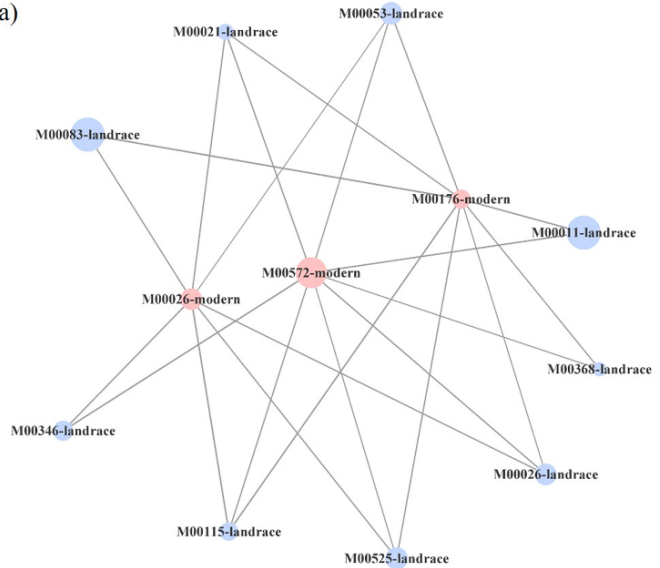

c)

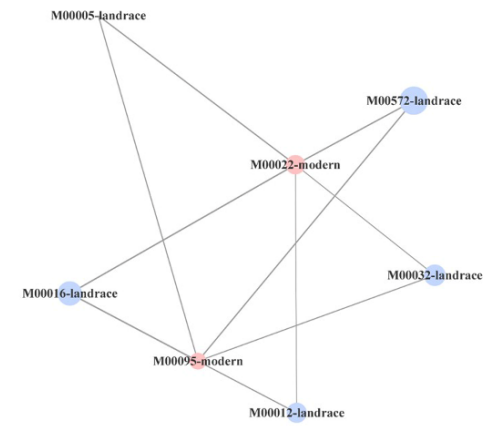

b)

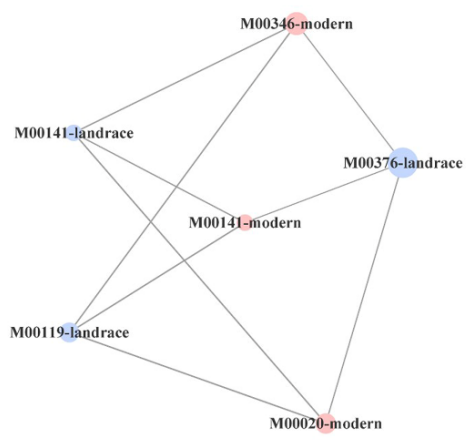

Figure 5. Three $(\mathbf{a}-\mathbf{c})$ most connected clusters in co-presence networks for the couple modern commercial cultivars (red):tomato landraces (blue). Node sizes reflect average relative abundance of each KEGG module. The line thickness is proportional to the edge weight. 


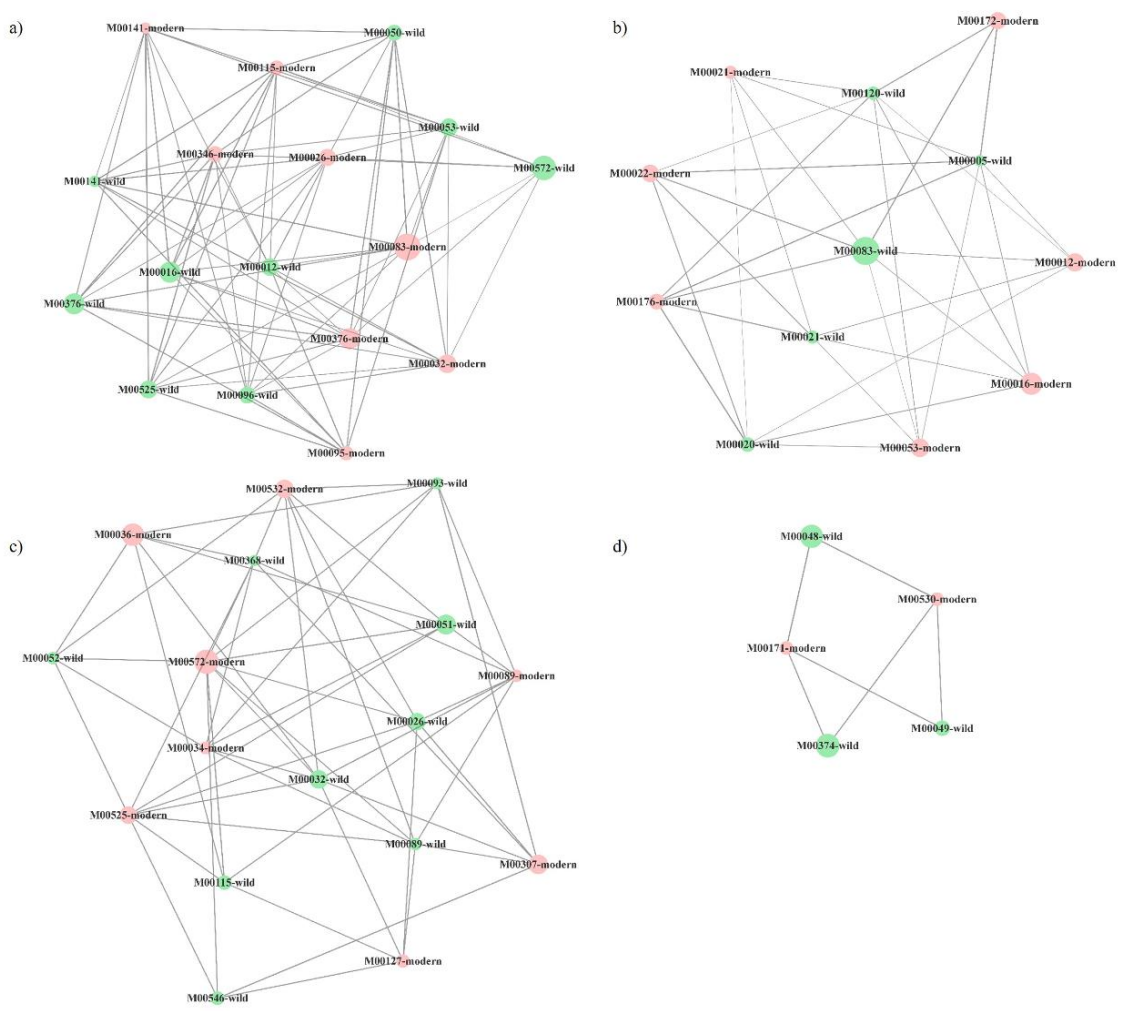

Figure 6. Four (a-d) most connected clusters in co-presence networks for the couple modern commercial cultivars (red):wild tomato relates species (green). Node sizes reflect average relative abundance of each KEGG module. The line thickness is proportional to the edge weight.

\section{Discussion}

Root traits selected during domestication were previously suggested to have a significant influence on the composition of the rhizosphere microbiome $[13,17]$. We found similar core bacterial microbiome members in tomato landraces and modern commercial cultivars, but detected small, though significant, differences in bacterial communities associated with both their rhizospheres and those of wild tomato relatives (Figure 1).

At family level, Gemmatimonadaceae (phylum Actinobacteria), Microbacteriaceae and Streptomycetaceae (Gemmatimonadetes) were represented less in the rhizosphere of wild tomato related species. At genera level, domestication gradually reduces the presence of the ubiquitous soil bacterium Pedobacter, the aromatic substrate metabolizer Rhodococcus and the alphaproteobacteria Skermanella and Microvirga, the latter considered a symbiotic nitrogen-fixing bacterium.

Previous studies highlighted the effect of plant species on the microbial composition and OTU abundance of the rhizosphere microbiome [5,18]. Domesticated crops often have shallow roots and shifts in traits such as leaf size and root architecture. Changes in these morphological traits results in increased litter quality, lower $\mathrm{C}: \mathrm{N}$ ratio and root exudate composition, which could influence microbial community composition $[2,9,19,20]$. In this study, bacterial diversity at the OTU level was found to remain virtually unchanged along the domestication gradient, although evenness levels were significantly lower in the rhizosphere of tomato wild relatives. Evenness refers to the similarity of OTU frequencies in bacterial populations. Even though species evenness and richness are complementary, no differences were observed in the latter; the number of soil bacterial phyla recruited by wild type crops was similar to other tomatoes. Nevertheless, evenness does not necessarily translate into optimal diversity; ecosystem functions at the bacterial community level are more important than the bacterial species. As several species in an ecosystem may fulfill a similar function (redundancy), their even distribution is not essential as long as 
the function itself remains active. However, a more even species distribution within a bacterial community is assumed to make the ecosystem more resilient, as the risk of losing an essential component of the functional network would be much lower.

Using metagenomic analysis, the functional potential of the bacterial community was predicted and the associated metabolic pathway network explored (Table 2). The levels of the global metabolic pathway for aromatic degradation were significantly higher in bacteria associated to accessions of tomato wild relatives. The modules belonging to this pathway catalyze reactions involving various polyphenols such as catechol. Humification is known to involve biotic and abiotic transformations of soil litter layer materials into mature humic substances, where catechol and o-quinones derived from biotic activity in humic substance synthesis play a fundamental role [21]. In addition, the increase in catechol promotes the formation of humic substances through abiotic reactions in the catechol-Maillard system [22]. Thus, the observed decrease in the degradation of aromatic compounds to catechol indicates a loss of degradation capacity due to cultivation. Organic matter and humic substances play an important role in improving soil fertility and structure, water retention capacity and $C$ sequestration in the environment [23], which diminishes along the domestication gradient. Another possible hypothesis is that plants affect microbial populations, and changes in environmental conditions, soils and cultivation techniques-with the gradual abandonment of organic materials in favor of agrochemicals-could reduce the degradation capacity of recalcitrant organic compounds associated with domestication and breeding. On the other hand, with respect to the carbon cycle, organic $\mathrm{C}$ taken up by microorganisms is partitioned into growth, metabolite excretion, and respiration [24]. We detected an increase in the Krebs cycle of wild tomato related species below-ground. After incorporation into the bacterial biomass, $\mathrm{C}$ is usually converted into stable organic matter or decomposed and respired as $\mathrm{CO}_{2}$ depending on the chemical recalcitrance and degree of protection of the organic matter [25].

In this context, it has been suggested that crop wild relatives establish beneficial interactions with microbes more frequently than domesticated cultivars [26]. Given the abandonment of some agricultural practices related to exogenous organic matter inputs and the preservation of endogenous $\mathrm{C}$, a concomitant loss of bacterial functions dealing with recalcitrant organic matter has been occurring for many years. It has also been evidenced that agronomic practices, such as tillage, irrigation and the use of other inputs such as pesticides and fertilizers influence the below-ground diversity and functions of soil microbes [27]. We therefore postulate that a loss in bacterial functions related to soil organic matter preservation occurs during tomato domestication.

A similar trend was detected in metabolic pathways related to biochemical cycles, such as the reduction in nitrates and sulphates and the formation of urea from purine metabolism. The decrease in these pathways that play a key role in plant growth could be attributed to the domestication process, or more precisely, to the emergence of modern commercial cultivars. Similar to the observations in the C-cycle, the increasing use of agrochemicals in modern agriculture may, in some way, be connected to the reduction on metabolic pathway levels caused by certain biochemical cycles.

Carbon fixation was more common in bacteria associated with modern commercial cultivars. This important process in soil carbon cycling is carried out by $\mathrm{CO}_{2}$-fixing and CO-oxidizing bacteria and can reduce atmospheric $\mathrm{CO}_{2}$ concentrations, thus indirectly mitigating global warming $[24,28,29]$. However, as no differences in the synthesis of ribulose 5 phosphate, an intermediary in the carbon fixation Calvin cycle, can be attributed to domestication, it is not possible to draw a clear picture of the effects of domestication on this ecosystem service.

On the other hand, pathways such as fatty acid and jasmonic acid biosynthesis were more commonly found in the rhizosphere of modern and landrace varieties. Fatty acids are involved in multiple functions, ranging from cell membrane constituents to cell signaling. Fatty acids have been used as indices of soil quality and even to describe food web connections [30], thus, positive feedback compared with their wild ancestors could be 
attributed to tomato crops. Jasmonic acid (JA) and its derivatives (collectively known as jasmonates) play an important role in regulating plant defenses against biotic stresses, and facilitating beneficial interactions between plants and microbes in the root zone [31,32]. JA signaling has been suggested to have evolved during land colonization by plants exposed to new biotic and abiotic stresses [33], and symbiotic relationships with microbes, including plant growth promoting bacteria and mycorrhizal fungi. Moreover, microbe induced systemic resistance to pathogens and pests involve JA signaling [34,35]. However, although JA production by bacteria and fungi in soil has been reported [36], its impact on plant-microbiome interactions remains unclear. Finally, regarding signalling, we detected a significant increase in the biosynthesis of gamma-aminobutyric acid (GABA) in wild tomato species compared to the groups that included cultivars. GABA is involved in inter-bacterial communication and interactions between bacteria and their host [37]. Furthermore, GABA production has been associated with bacterial overcoming of environmental stress [38].

Overall, these findings highlight the influence of tomato domestication on some molecular pathways of the associated soil bacteria, although all ancestral functional characteristics of bacteria have been conserved across time. However, we wonder whether there is a pattern of bacterial functional abundance associated with the tomato soil related to the domestication degree. To shed some light on this point, we calculated interactions between functional units of gene sets in metabolic pathways, which may help to address the question of how microbial genes work together to support specific microbiome functions [39]. In this study, we assessed pairwise relationships between bacterial functional units based on metagenomic sequencing of bacteria growing on tomato plants along a domestication gradient The highest connectance levels in bacterial communities were found in landraces:wild pairs due to an increase in network density as measured by the higher average number of connections established expressed by the average number of neighbors (Table 3). In addition, the increased connectance in the landraces:wild pairing with respect to the other two pairs was related to the decrease in the characteristic path length, defined as the average number of steps along the shortest paths for all possible pairs of network nodes. These changes suggest an intensification of microbial connectance relative to the pairs modern:landraces and modern:wild. Finally, an increase in the pair modern:landraces regarding the network radius and diameter measuring the longest of all the shortest calculated paths in the network, suggests a decrease in module-pathway connectance.

Highly connected clusters, or sets of nodes most of which are connected with one another, were then explored (Figures 4-6). Again, the highest connectance was detected for the pair landraces:wild varieties and nodes representing the same module in the two different types of tomato were recovered in a single cluster. For the rest of the pairs, even if they shared the same number of common modules, they were recovered in two or three different clusters. Overall, the above results suggest that certain functional groups such as the synthesis of certain amino acids or carbohydrate metabolism tend to be more evolutionarily conserved in bacterial communities associated with tomato landraces than those of modern varieties. However, we also found that most of the metabolic routes of bacteria associated to either landraces or modern cultivars with those associated to their ancestors were different. In this scenario, a possible process of divergent evolution in tomato lines, that is, the process by which groups of the same common ancestor evolve and accumulate differences in response to changes in both environmental conditions and biotic factors, could be debated. Nevertheless, further investigation is needed to clarify how tomato domestication has driven specific bacterial functions in root-associated soil.

\section{Materials and Methods}

\subsection{Field Experiment}

Seeds of 27 Solanum lycopersicum Mill., S. habrochaites and S. pimpinellifolium accessions were selected from La Mayora Institute of Subtropical and Mediterranean Horticulture (IHSM-UMA-CSIC) germplasm bank. Seeds were germinated and ten one-month-old seedlings per variety $(n=270)$ were randomly sown in an experimental field of La Mayora 
IHSM (Málaga, Spain; 36.77 $\mathrm{N}, 4.04^{\circ} \mathrm{W}$ ), ) on 19th April 2018 in a Eutric Regosol soil [40]. They were grown until 16th July 2018. Just after transplanting, plants were watered with $15: 15: 15$ solution ( $15 \%$ nitrogen, $15 \%$ phosphorus and $15 \%$ potassium) during $30 \mathrm{~min}$ adding-up a volume of 41 per plant. During the course of the experiment, watering consisted of $30 \mathrm{~min}$ of water twice in a week (Mondays and Fridays) [41]. At harvest, the soil attached to the main and secondary roots was taken by shaking, placed in separate plastic bags, and kept at $4{ }^{\circ} \mathrm{C}$. Then, samples from each variety were pooled, ground together using a mortar and pestle, sieved twice (2-mm mesh) and immediately stored at $-20{ }^{\circ} \mathrm{C}$ until molecular analyses were performed.

In this study, cluster assays of the 27 tomato accessions, based on their degree of domestication, were carried out: (1) wild tomato species (accessions NR0407, NR1021, NR0136, NR0699, NR0937), (2) tomato landraces (accessions NR0025, NR0006, NR0044, NR0213, NR0275, NR0237, NR0469, NR0166, NR0063, NR0705, NR0612), and (3) modern commercial cultivars (accessions ABL104, ANL101, NR0071, NR0816, NR0080, NR1080, NR0504. COM1, COM2, COM3 and COM4 cultivars, which are protected under plant variety rights, have no accession number).

\subsection{Chemical Characteristics of the Soil}

Air-dried rhizosphere soil samples were used to determinate chemical properties. Total $\mathrm{N}$ and SOC were determined with the aid of the Leco-TruSpec $\mathrm{CN}$ elemental analyzer (LECO Corp., St Joseph, MI, USA). Total mineral content was determined by the digestion method with $\mathrm{HNO} 365 \%: \mathrm{HCl} 35 \%$ (1:3; v:v) followed by analysis using inductively coupled plasma optical emission spectrometry (ICP-OES) (ICP 720-ES, Agilent, Santa Clara, CA, USA). Detailed information on soil characteristics is given in Supplementary Material Table S2.

\subsection{Molecular Analyses of Soil Bacteria}

DNA was extracted from eight $1 \mathrm{~g}$ aliquots for each root-associated soil sample using the bead-beating method with the aid of a PowerSoil ${ }^{\circledR}$ DNA Isolation Kit (MoBio Laboratories, Solana Beach, CA, USA) according to the manufacturer's instructions. For each variety, two replicates were prepared by pooling four extractions and concentrating them at $35^{\circ} \mathrm{C}$ to a final volume of $20 \mu \mathrm{L}$ using a Savant Speedvac ${ }^{\circledR}$ concentrator (Fisher Scientific, Madrid, Spain). The V3-V4 hypervariable regions (ProV3V4 primers 5' CCTACGGGNBGCASCAG $3^{\prime}$ and $5^{\prime}$ GACTACNVGGGTATCTAATCC $3^{\prime}$ [42,43]) of the 16S rRNA gene were used to characterize the bacterial communities of the two replicates per sample using the Illumina MiSeq platform $(2 \times 250$ nucleotide paired-end protocol $)$ at the genomic facilities of the López-Neyra Institute of Parasitology and Biomedicine (IPBLN-CSIC). Blockers were used to minimize amplification of mitochondria and chloroplasts. Raw sequences were preprocessed using the SEED2 platform [44] by first merging forward and reverse sequences. Quality filtering excluded sequences containing ambiguous bases $(\mathrm{N})$ and those with a quality score of less than 30 . Primers were removed and sequences trimmed to 400 bp length. The sequences were then clustered using the UPARSE method [45]: Operational Taxonomic Unit (OTU) radius set to $3 \%$ and sequence similarity to $97 \%$. Singletons and chimeric sequences were removed. Taxonomic assignment of OTUs was performed using the classify.seqs algorithm in Mothur software (University of Michigan, Detroit, MI, USA) against the SILVA v132 database, after which no archaea were detected in the samples [46,47]. An abundance sample x OTU matrix was generated using OTU reads as a proxy of abundance using the Marker Data Profiling module in the MicrobiomeAnalyst tool (https: / / www.microbiomeanalyst.ca / accessed on 2 August 2021). The most abundant sequence per OTU was selected as representative. Rarefaction curves were visualized using MicrobiomeAnalyst to confirm that all samples reached a plateau $[48,49]$. 


\subsection{Predictive Metagenomics Profiling}

To determine the potential functional metabolic capabilities of soil bacterial communities, we used Tax4fun, an open-source $R$ package, which predicts the functional capabilities of these communities based on $16 \mathrm{~S}$ datasets. Tax4Fun is applicable to output obtained from the SILVAngs web server [50]. Tax4fun was implemented in Shotgun Data Profiling (SDP) module of MicrobiomeAnalyst to predict functional pathways based on Kyoto Encyclopedia of Gene and Genome (KEGG, https:/ / www.kegg.jp/ accessed on 2 August 2021) annotations [51,52]. KEGG functional annotations were based on modules, i.e., functional units of gene sets in the KEGG metabolic pathways database that can be linked to specific metabolic capacities and other phenotypic features [48].

\subsection{Functional Networks}

Similarities on functional profiles across tomato types were studied by looking for correlations in the abundance of modules. CoNet plug-in method [53] in Cytoscape software v.3.8.2 [54] was used to visualize these relationships by building co-occurrence networks. Thus, two nodes representing the same module in different tomato types should be connected in the case that both tomatoes have a similar pattern of abundance for that module. Thus, building networks by tomato type pairs gives an idea of the conservation of modules across domestication (i.e., the number of links between the same module in different tomato types). Co-occurrence networks were constructed based on the identification of significant positive associations, that is, co-presences of functional units in the tomato root-associated microbiome. Due to the different number of samples/tomato varieties in each domestication type, for arranging the construction of network, the number of samples in each domestication type was adjusted to the tomato type with the least number. The selection and order of samples was arranged randomly. This analysis was repeated 5 times by shuffling the input sample order to avoid spurious results. To run the analysis, KEGG modules having less than 20 reads were discarded from the analyses. KEGG module abundance was normalized by sample. A total of 2000 permutations were set up by keeping edge number constant. The significance of co-presences were evaluated by a combination of Spearman and Pearson correlations and Bray-Curtis dissimilarity (see e.g., [52,55], corrected for multiple testing using Bonferroni). Finally, the MCODE Cytoscape plugin [56] with default settings was then used to detect highly connected network modules. Only modules with an MCODE score greater than 2.0 were retained for analysis [39].

\subsection{Statistical Analyses}

OTU abundance information was normalized to the abundance value of the sample with the least number of sequences. Alpha diversity indices generated by SEED2 were used to compare bacterial richness and diversity in tomato accessions. Statistically significant differences in alpha diversity, the bacterial composition of the group of tomato varieties and predictive metagenomics profiling data were evaluated using generalized lineal model (GLM) with degree of domestication as fixed factor. We checked fixed factors for significance with Wald test from car package [57] and multiple comparisons between levels of the fixed factor were tested using Tukey's test with the package lsmeans and emmeans [58]. For each model, residuals were examined for model validation. Beta diversity, or species complexity differences between groups of tomato varieties, was determined by linear discriminant analysis (LDA) effect size (LEfSe) using the MicrobiomeAnalyst web server. Taxa with an LDA score $>4$ were considered important biomarkers of each group given that a $p$ value $<0.05$ indicates significant differences between groups. Data were analyzed using $R$ version 3.6.3 [59] and $R$ Studio version 1.1.456 [60].

\section{Conclusions}

In our study we found that core bacterial microbiome is similar between tomato landraces and modern commercial cultivars with small differences with wild tomato. These 
findings highlight the influence of the host genotype on the potential functions of soil bacterial communities. Furthermore, we found that differences in eight biological metabolic pathways between wild tomatoes compared with tomato landraces and modern commercial. Thus, we conclude that all ancestral functional characteristics of bacteria have been conserved across time. In the light of these results, it becomes apparent that the capacity of soil bacteria to provide ecosystem services is affected by agronomic practices linked to the domestication process, particularly those related to the preservation of soil organic matter. We also assayed the relationships between functional units of bacteria growing on tomato plants along a domestication gradient, finding the highest levels of connection between bacterial communities driven by tomato landraces and their wild ancestors.

Supplementary Materials: The following are available online at https:/ / www.mdpi.com/article / 10.3390/plants10091942/s1, Table S1: Abundance of predicted functions related to root-associated soil bacteria in the 27 tomato accessions. Table S2: Chemical characteristics of the soil. Table S3: Tab-delimited taxonomy tables.

Author Contributions: Conceptualization, E.B., E.d.1.P. and L.S.; methodology, L.S., B.M., V.F. and R.A.H.; software, L.S., Á.L.-G., R.A.H. and E.B.; validation, E.B., R.A.H., L.S. and Á.L.-G.; formal analysis, E.B., L.S., R.A.H. and Á.L.-G.; investigation, E.B., L.S. and Á.L.-G.; resources, E.B., E.d.1.P., V.F. and M.J.P.; data curation, L.S., Á.L.-G., B.M., R.A.H. and E.B.; writing-original draft preparation, E.B., Á.L.-G. and L.S.; writing-review and editing, R.A.H., B.M., E.d.l.P. and M.J.P.; supervision, E.B. and M.J.P.; project administration; E.B.; funding acquisition, E.B. and M.J.P. All authors have read and agreed to the published version of the manuscript.

Funding: This research has received funding from the European Union's Horizon 2020 Research and Innovation programme under Grant agreement No 765290.

Institutional Review Board Statement: Not applicable.

Informed Consent Statement: Not applicable.

Data Availability Statement: All raw Illumina sequence data were deposited in the Sequence Read Archive (SRA) service of the European Bioinformatics Institute (EBI) database (BioProject ID: PRJNA693664).

Acknowledgments: Rafael Alcalá Herrera thanks the Junta de Andalucía (Spain) for funding his research at the EEZ-CSIC with a postdoctoral contract (Project P12-AGR-1419).

Conflicts of Interest: The authors declare no conflict of interest.

\section{References}

1. Reinhold-Hurek, B.; Bünger, W.; Burbano, C.S.; Sabale, M.; Hurek, T. Roots shaping their microbiome, global hotspots for microbial activity. Annu. Rev. Phytopathol. 2015, 53, 403-424. [CrossRef] [PubMed]

2. Cordovez, V.; Dini-Andreote, F.; Carrión, V.J.; Raaijmakers, J.M. Ecology and Evolution of Plant Microbiomes. Annu. Rev. Microbiol. 2019, 73, 69-88. [CrossRef] [PubMed]

3. Wall, D.H.; Bardgett, R.D.; Behan-Pelletier, V.; Herrick, J.E.; Jones, H.; Ritz, K.; Six, J.; Strong, D.R.; van der Putten, W.H. Soil Ecology and Ecosystem Services; Oxford University Press: Oxford, UK, 2012.

4. Houlden, A.; Timms-Wilson, T.M.; Day, M.J.; Bailey, M.J. Influence of plant developmental stage on microbial community structure and activity in the rhizosphere of three field crops. FEMS Microbiol. Ecol. 2008, 65, 193-201. [CrossRef]

5. Lei, S.; Xu, X.; Cheng, Z.; Xiong, J.; Ma, R.; Zhang, L.; Yang, X.; Zhu, Y.; Zhang, B.; Tian, B. Analysis of the community composition and bacterial diversity of the rhizosphere microbiome across different plant taxa. Microbiol. Open 2019, 8, e00762. [CrossRef]

6. Compant, S.; Samad, A.; Faist, H.; Sessitsch, A. A review on the plant microbiome, ecology; functions; and emerging trends in microbial application. J. Adv. Res. 2019, 19, 29-37. [CrossRef]

7. Liu, H.; Brettell, L.E.; Qiu, Z.; Singh, B.K. Microbiome-mediated stress resistance in plants. Trends Plant. Sci. 2020, 25, 733-743. [CrossRef]

8. Trivedi, P.; Leach, J.E.; Tringe, S.G.; Sa, T.; Singh, B.K. Plant-microbiome interactions, from community assembly to plant health. Nat. Rev. Microbiol. 2020, 18, 607-621. [CrossRef]

9. Pérez-Jaramillo, J.E.; Mendes, R.; Raaijmakers, J.M. Impact of plant domestication on rhizosphere microbiome assembly and functions. Plant. Mol. Biol. 2016, 90, 635-644. [CrossRef] [PubMed]

10. Schmidt, J.E.; Bowles, T.M.; Gaudin, A.C.M. Using ancient traits to convert soil health into crop yield, impact of selection on maize root and rhizosphere function. Front. Plant. Sci. 2016, 7, 1-11. [CrossRef] 
11. Iannucci, A.; Fragasso, M.; Beleggia, R.; Nigro, F.; Papa, R. Evolution of the Crop Rhizosphere, Impact of Domestication on Root Exudates in Tetraploid Wheat (Triticum turgidum L.). Front. Plant. Sci. 2017, 8, 2124. [CrossRef]

12. Pérez-Jaramillo, J.E.; Carrión, V.J.; de Hollander, M.; Raaijmakers, J.M. The wild side of plant microbiomes. Microbiome 2018, 6, 143. [CrossRef]

13. Shenton, M.; Iwamoto, C.; Kurata, N.; Ikeo, K. Effect of Wild and Cultivated Rice Genotypes on Rhizosphere Bacterial Community Composition. Rice 2016, 9, 42. [CrossRef]

14. Carrillo, J.; Ingwell, L.L.; Li, X.; Kaplan, I. Domesticated tomatoes are more vulnerable to negative plant-soil feedbacks than their wild relatives. J. Ecol. 2019, 107, 1753-1766. [CrossRef]

15. Terrazas, R.A.; Balbirnie-Cumming, K.; Morris, J.; Hedley, P.E.; Russell, J.; Paterson, E.; Baggs, E.M.; Fridman, E.; Bulgarelli, D.A. A footprint of plant eco-geographic adaptation on the composition of the barley rhizosphere bacterial microbiota. Sci. Rep. 2020, 10, 12916. [CrossRef]

16. Jia, Y.; Whalen, J.K. A new perspective on functional redundancy and phylogenetic niche conservatism in soil microbial communities. Pedosphere 2020, 30, 18-24. [CrossRef]

17. Spor, A.; Roucou, A.; Mounier, A.; Bru, D.; Breuil, M.C.; Fort, F.; Vile, D.; Roumet, P.; Philippot, L.; Violle, C. Domestication-driven changes in plant traits associated with changes in the assembly of the rhizosphere microbiota in tetraploid wheat. Sci. Rep. 2020, 10, 12234. [CrossRef] [PubMed]

18. Mendes, R.; Garbeva, P.; Raaijmakers, J.M. The rhizosphere microbiome, significance of plant beneficial; plant pathogenic; and human pathogenic microorganisms. FEMS Microbiol. Rev. 2013, 37, 634-663. [CrossRef] [PubMed]

19. Martínez-Romero, E.; Aguirre-Noyola, J.L.; Taco-Taype, N.; Martínez-Romero, J.; Zuñiga-Dávila, D. Plant microbiota modified by plant domestication. Syst. Appl. Microbiol. 2020, 43, 126106. [CrossRef]

20. Milla, R.; García-Palacios, P.; Matesanz, S. Looking at past domestication to secure ecosystem services of future croplands. J. Ecol. 2017, 105, 885-889. [CrossRef]

21. Stevenson, F.J. Humus Chemistry, Genesis, Composition, Reactions, 2nd ed.; John Wiley and Sons: New York, NY, USA, 1994.

22. Zhang, Y.; Yue, D.; Ma, H. Darkening mechanism and kinetics of humification process in catechol-Maillard system. Chemosphere 2015, 130, 40-45. [CrossRef]

23. Tiessen, H.; Cuevas, E.; Chacon, P. The role of soil organic matter in sustaining soil fertility. Nature 1994, 371, 783-785. [CrossRef]

24. Gougoulias, C.; Clark, J.M.; Shaw, L.J. The role of soil microbes in the global carbon cycle, tracking the below-ground microbial processing of plant-derived carbon for manipulating carbon dynamics in agricultural systems. J. Sci. Food Agric. 2014, 94, 2362-2371. [CrossRef] [PubMed]

25. Six, J.; Frey, S.D.; Thiet, R.K.; Batten, K.M. Bacterial and Fungal Contributions to Carbon Sequestration in Agroecosystems. Soil Sci. Soc. Am. J. 2006, 70, 555-569. [CrossRef]

26. Pérez-Jaramillo, J.E.; de Hollander, M.; Ramírez, C.A.; Mendes, R.; Raaijmakers, J.M.; Carrión, V.J. Deciphering rhizosphere microbiome assembly of wild and modern common bean (Phaseolus vulgaris) in native and agricultural soils from Colombia Microbiome 2019, 7, 114. [CrossRef]

27. Vries, F.; Wallenstein, M.D. Below-ground connections underlying above-ground food production: A framework for optimising ecological connections in the rhizosphere. J. Ecol. 2017, 105, 913-920. [CrossRef]

28. Cavicchioli, R.; Ripple, W.J.; Timmis, K.N.; Azam, F.; Bakken, L.R.; Baylis, M.; Behrenfeld, M.J.; Boetius, A.; Boyd, P.W.; Classen, A.T.; et al. Scientists' warning to humanity, microorganisms and climate change. Nat. Rev. Microbiol. 2019, 17, 569-586. [CrossRef] [PubMed]

29. Lynn, T.M.; Ge, T.; Yuan, H.; Wei, X.; Wu, X.; Xiao, K.; Kumaresan, D.; Yu, S.S.; Wu, J.; Whiteley, A.S.; et al. Soil Carbon-Fixation Rates and Associated Bacterial Diversity and Abundance in Three Natural Ecosystems. Microb. Ecol. 2017, 73, 645-657. [CrossRef] [PubMed]

30. de Carvalho, C.; Caramujo, M.J. The Various Roles of Fatty Acids. Molecules 2018, 23, 2583. [CrossRef] [PubMed]

31. Pieterse, C.M.; Leon-Reyes, A.; Van der Ent, S.; Van Wees, S.C. Networking by small-molecule hormones in plant immunity. Nat. Chem. Biol. 2009, 5, 308-316. [CrossRef]

32. Bari, R.; Jones, J.D. Role of plant hormones in plant defence responses. Plant. Mol. Biol. 2009, 69, 473-488. [CrossRef]

33. Kenrick, P.; Crane, P. The origin and early evolution of plants on land. Nature 1997, 389, 33-39. [CrossRef]

34. Pozo, M.J.; López-Ráez, J.A.; Azcón-Aguilar, C.; García-Garrido, J.M. Phytohormones as integrators of environmental signals in the regulation of mycorrhizal symbioses. New Phytol. 2015, 205, 1431-1436. [CrossRef] [PubMed]

35. Gruden, K.; Lidoy, J.; Petek, M.; Podpečan, V.; Flors, V.; Papadopoulou, K.K.; Pappas, M.L.; Martinez-Medina, A.; Bejarano, E.; Biere, A.; et al. Ménage à Trois, Unraveling the Mechanisms Regulating Plant-Microbe-Arthropod Interactions. Trends Plant. Sci. 2020, 25, 1215-1226. [CrossRef] [PubMed]

36. Eng, F.; Marin, J.E.; Zienkiewicz, K.; Gutiérrez-Rojas, M.; Favela-Torres, E.; Feussner, I. Jasmonic acid biosynthesis by fungi, derivatives; first evidence on biochemical pathways and culture conditions for production. Peer] 2021, 9, e10873. [CrossRef] [PubMed]

37. Dagorn, A.; Chapalain, A.; Mijouin, L.; Hillion, M.; Duclairoir-Poc, C.; Chevalier, S.; Taupin, L.; Orange, N.; Feuilloley, M.G. Effect of GABA, a bacterial metabolite; on Pseudomonas fluorescens surface properties and cytotoxicity. Int. J. Mol. Sci. 2013, 14, 12186-12204. [CrossRef] 
38. Somorin, Y.; Abram, F.; Brennan, F.; O’Byrne, C. The General Stress Response Is Conserved in Long-Term Soil-Persistent Strains of Escherichia coli. Appl. Environ. Microbiol. 2016, 82, 4628-4640. [CrossRef] [PubMed]

39. Li, L.; Wang, Z.; He, P.; Ma, S.; Du, J.; Jiang, R. Construction and Analysis of Functional Networks in the Gut Microbiome of Type 2 Diabetes Patients. Genom. Proteom. Bioinform. 2016, 14, 314-324. [CrossRef]

40. FAO. World Reference Base for Soil Resources 2014: International Soil Classification System for Naming Soils and Creating Legends for Soil Maps; Food and Agriculture Organization of the United Nations: Roma, Italy, 2015.

41. Ferrero, V.; Baeten, L.; Blanco-Sanchez, L.; Planello, R.; Diaz-Pendon, J.A.; Rodríguez-Echeverría, S.; Haegeman, A.; de la Peña, E. Complex patterns in tolerance and resistance to pests and diseases underpin the domestication of tomato. New Phytol. 2020, 226, 254-266. [CrossRef] [PubMed]

42. Takahashi, S.; Tomita, J.; Nishioka, K.; Hisada, T.; Nishijima, M. Development of a prokaryotic universal primer for simultaneous analysis of Bacteria and Archaea using next-generation sequencing. PLoS ONE 2014, 9, e105592. [CrossRef]

43. Lundberg, D.S.; Yourstone, S.; Mieczkowski, P.; Jones, C.D.; Dangl, J.L. Practical innovations for high-throughput amplicon sequencing. Nat. Methods 2013, 10, 999-1002. [CrossRef]

44. Vetrovský, T.; Baldrian, P.; Morais, D. SEED 2, a user-friendly platform for amplicon high-throughput sequencing data analyses. Bioinformatics 2018, 34, 2292-2294. [CrossRef]

45. Edgar, R.C. UPARSE, highly accurate OTU sequences from microbial amplicon reads. Nat. Methods 2013, 10, 996. [CrossRef] [PubMed]

46. Schloss, P.D.; Westcott, S.L.; Ryabin, T.; Hall, J.R.; Hartmann, M.; Hollister, E.B.; Lesniewski, R.A.; Oakley, B.B.; Parks, D.H.; Robinson, C.J.; et al. Introducing mothur, open-source; platform-independent; community-supported software for describing and comparing microbial communities. Appl. Environ. Microbiol. 2009, 75, 7537-7541. [CrossRef] [PubMed]

47. Quast, C.; Pruesse, E.; Yilmaz, P.; Gerken, J.; Schweer, T.; Yarza, P.; Peplies, J.; Glöckner, F.O. The SILVA ribosomal RNA gene database project, improved data processing and web-based tools. Nucleic Acids Res. 2013, 41, 590-596. [CrossRef]

48. Chong, J.; Liu, P.; Zhou, G.; Xia, J. Using MicrobiomeAnalyst for comprehensive statistical; functional; and meta-analysis of microbiome data. Nat. Protoc. 2020, 15, 799-821. [CrossRef]

49. Dhariwal, A.; Chong, J.; Habib, S.; King, I.L.; Agellon, L.B.; Xia, J. MicrobiomeAnalyst, a web-based tool for comprehensive statistical; visual and meta-analysis of microbiome data. Nucleic Acids Res. 2017, 45, 180-188. [CrossRef]

50. Aßhauer, K.P.; Wemheuer, B.; Daniel, R.; Meinicke, P. Tax4Fun, predicting functional profiles from metagenomic $16 \mathrm{~S}$ rRNA data. Bioinformatics 2015, 31, 2882-2884. [CrossRef]

51. Breiman, L. Random forests. Mach. Learn. 2001, 45, 5-32. [CrossRef]

52. Segata, N.; Izard, J.; Waldron, L.; Gevers, D. Metagenomic biomarker discovery and explanation. Genome Biol. 2011, 12, R60. [CrossRef]

53. Faust, K.; Raes, J. CoNet app, inference of biological association networks using Cytoscape. F1000Research 2016, 5, 1519. [CrossRef] [PubMed]

54. Shannon, P.; Markiel, A.; Ozier, O.; Baliga, N.S.; Wang, J.T.; Ramage, D.; Amin, N.; Schwikowski, B.; Ideker, T. Cytoscape, a software environment for integrated models of biomolecular interaction networks. Genome Res. 2003, 13, 2498-2504. [CrossRef] [PubMed]

55. Weiss, S.; Van Treuren, W.; Lozupone, C.; Faust, K.; Friedman, J.; Deng, Y.; Xia, L.C.; Xu, Z.Z.; Ursell, L.; Alm, E.J.; et al. Correlation detection strategies in microbial data sets vary widely in sensitivity and precision. ISME J. 2016, 10, 1669-1681. [CrossRef] [PubMed]

56. Bader, G.D.; Hogue, C.W. An automated method for finding molecular complexes in large protein interaction networks. BMC Bioinform. 2003, 4, 2. [CrossRef] [PubMed]

57. Fox, J.; Weisberg, S. An R Companion to Applied Regression, 3rd ed.; Sage: Thousand Oaks, CA, USA, 2019.

58. Lenth, R.V. Least-squares means, The R package lsmeans. J. Stat. Soft 2016, 69, 1-33. [CrossRef]

59. R Development Core Team. R, A Language and Environment for Statistical Computing; R Development Core Team: Vienna, Austria, 2017.

60. RStudio Team. RStudio, Integrated Development Environment for R; RStudio Team: Boston, MA, USA, 2016. 